\title{
Natural Extension of the Schrödinger Equation to Quasi-Relativistic Speeds
}

\author{
Luis Grave de Peralta1,2 \\ ${ }^{1}$ Department of Physics and Astronomy, Texas Tech University, Lubbock, TX, USA \\ ${ }^{2}$ Nano Tech Center, Texas Tech University, Lubbock, TX, USA \\ Email: luis.grave-de-peralta@ttu.edu
}

How to cite this paper: Grave de Peralta, L. (2020) Natural Extension of the Schrödinger Equation to Quasi-Relativistic Speeds. Journal of Modern Physics, 11, 196-213. https://doi.org/10.4236/jmp.2020.112012

Received: January 10, 2020

Accepted: February 3, 2020

Published: February 6, 2020

Copyright (c) 2020 by author(s) and Scientific Research Publishing Inc. This work is licensed under the Creative Commons Attribution International License (CC BY 4.0).

http://creativecommons.org/licenses/by/4.0/

\begin{abstract}
A Schrödinger-like equation for a single free quantum particle is presented. It is argued that this equation can be considered a natural relativistic extension of the Schrödinger equation for energies smaller than the energy associated to the particle's mass. Some basic properties of this equation: Galilean invariance, probability density, and relation to the Klein-Gordon equation are discussed. The scholastic value of the proposed Grave de Peralta equation is illustrated by finding precise quasi-relativistic solutions for the infinite rectangular well and the quantum rotor problems. Consequences of the non-linearity of the proposed equation for the quantum superposition principle are discussed.
\end{abstract}

\section{Keywords}

Quantum Mechanics, Schrödinger Equation, Klein-Gordon Equation, Relativistic Quantum Mechanics

\section{Introduction}

Since the discovery of the quantum wave mechanics by Erwin Schrödinger in 1925, the Schrödinger equation has been often used for introducing the fundamentals of quantum mechanics [1] [2] [3] [4] [5]. The one-dimensional Schrödinger equation for a free particle with mass $m$ is given by the following equation [1] [2] [3] [4] [5]:

$$
i \hbar \frac{\partial}{\partial t} \psi_{\text {Sch }}(x, t)=-\frac{\hbar^{2}}{2 m} \frac{\partial^{2}}{\partial x^{2}} \psi_{S c h}(x, t) .
$$

where $\hbar$ is the Plank constant $(h)$ divided by $2 \pi$. However, the Schrödinger equation is not Lorentz invariant but Galilean invariant [6]; therefore, a relativistic 
quantum mechanics cannot be based on Equation (1). A fully relativistic quantum theory requires to be funded on equations that are valid for any two observers moving respect to each other at constant velocity. In contrast, the Galilean invariance of Equation (1) means that two such observers will only agree in the adequacy of Equation (1) for describing the movement of a massive free quantum particle when the relative speed between the observers $\left(V_{o}\right)$ is much smaller than the speed of the light in the vacuum (c). In practice, this is not a terrible limitation of the Schrödinger equation because up to today humans have been only able to travel at speeds much smaller than $c$. This is one of the principal reasons why the Schrödinger equation is still relevant almost 100 years after its discovery. However, as it will be discussed in Section 2, there is another important limitation of Equation (1): it describes a particle in which linear momentum $(p)$ and kinetic energy $(K)$ are related by a classical relation that is not valid at relativistic speeds [1] [2] [3] [6]. The famous relativistic equation $E_{m}=m c^{2}$, where $E_{m}$ is the energy associated to the mass of a particle [7] [8], implies the equivalence between mass and energy. This equivalence has profound implications for the formulation of any relativistic quantum mechanics theory. When the kinetic energy of a free particle with mass $m$ equals the energy associated to the mass of the particle, i.e., $K=m c^{2}$, a second particle with the same mass can be created from the kinetic energy of the original particle; therefore, the number of particles may not be conserved in a fully relativistic quantum theory [2] [8] [9]. A common argument used for guiding the search for the correct Lorentz invariant basic equation of a relativistic quantum mechanics is that in such equation the time and spatial variables should appear on equal footing as it happens in the Lorentz transformations [8] [9]. For instance, in contrast to Equation (1), in the Lorentz invariant Klein-Gordon equation does not appear the first partial derivative respect to time but the second one as shown in Equation (2), which is the Klein-Gordon equation for free particle [8] [9]:

$$
\frac{1}{c^{2}} \frac{\partial^{2}}{\partial t^{2}} \psi_{K G}(x, t)=\frac{\partial^{2}}{\partial x^{2}} \psi_{K G}(x, t)-\frac{m^{2} c^{2}}{\hbar^{2}} \psi_{K G}(x, t) .
$$

Unfortunately, Equation (2) does not formally look at all like Equation (1), thus masking how the Klein-Gordon equation becomes the Schrödinger equation when the particle moves at speeds $(V)$ much smaller than $c$. Moreover, there are solutions of Equation (2) with unwanted properties like superluminal phase velocity, negatives energies, and associated with negative probabilities [8] [9]. In Section 2, the consequences of an intriguing natural extension of the Schrödinger equation to quasi-relativistic speeds are explored. The term "quasi-relativistic" is used in this work as meaning a particle moving at so large speeds that it is necessary to use the correct relativistic relation between $p$ and $K$ but still the number of particles is constant because $K<m c^{2}$. The following equation is the center of attention here:

$$
i \hbar \frac{\partial}{\partial t} \psi(x, t)=-\frac{\hbar^{2}}{\left(\gamma_{V}+1\right) m} \frac{\partial^{2}}{\partial x^{2}} \psi(x, t) .
$$


where:

$$
\gamma_{V}=\frac{1}{\sqrt{1-\frac{V^{2}}{c^{2}}}} .
$$

Clearly, the Grave de Peralta equation (Equation (3)) exactly coincides with the Schrödinger equation (Equation (1)) when $V \ll c$. As it will be discussed in Section 3, the formal similitude between Equation (3) and Equation (1) immediately suggest that Equation (3) may be Galilean invariant and that probabilities can be associated to $\psi$ in the same way that it is done for $\psi_{\text {Sch }}$ [1] [2] [3] [4]. However, Equation (3) describes the movement of a massive free quantum particle which momentum and kinetic energy are related by the correct relativistic relation. Therefore, Equation (3) extent the range of applications of the Schrödinger equation to quasi-relativistic speeds. Moreover, the formal similitude between Equation (3) and Equation (1) provides a simple approach for obtaining quasi-relativistic corrections to the solutions of the Schrödinger equation, for a whole class of problems where the square of the particle speed $\left(V^{2}\right)$ is constant. Two interesting examples illustrating this point are presented in Sections 4 and 6. In both instances, explicit quasi-relativistic solutions of Equation (3) can be found with no more complexity than in standard textbook examples of solvable Schrödinger equation problems [1] [2] [3] [4] [5]. This illustrates the scholastic value of the Grave de Peralta equation for introducing learners to the intricacies of the fully relativistic quantum mechanics and quantum fields theory. In addition, it is demonstrated in this work that a plane wave solution of Equation (3) is subluminal and that this solution is related to a plane wave solution of Equation (2) by the following relationship:

$$
\psi(x, t)=\psi_{K G}(x, t) \mathrm{e}^{i w_{m} t}, w_{m}=\frac{m c^{2}}{\hbar} .
$$

where:

$$
\begin{aligned}
& \psi(x, t)=\mathrm{e}^{\frac{i}{\hbar}(p x-K t)}, \\
& \psi_{K G}(x, t)=\mathrm{e}^{\frac{i}{\hbar}(p x-E t)} .
\end{aligned}
$$

The plane waves $\psi$ and $\psi_{K G}$ in Equation (6) are solutions of Equations (3) and (2), respectively. $E, K$ and $p$ are the relativistic total and kinetic energy and the linear momentum of a free particle, respectively [7] [8]. It is worth noting that two solutions of Equation (3) corresponding to two different particle's speeds are not simultaneously solution of the same equation but solutions of two slightly different equations only differing in the value of $\gamma_{v}$. Even when the full discussion of this topic is outside of the scope of this work, due to its relevance, the implications of the non-linearity of Equation (3) for the quantum mechanics superposition principle are briefly discussed in Section 5. Finally, the conclusions of this work are given in Section 7. 


\section{Schrödinger Equation Extension to Quasi-Relativistic Speeds}

Formally, Equation (1) can be obtained from the classical relation between $K$ and $p$ for a free particle when $V \ll c$ [1] [2] [3] [6]:

$$
K=\frac{p^{2}}{2 m}, p=m V .
$$

Then, Equation (1) is obtained by substituting $K$ and $p$ by the following energy and momentum quantum operators [1] [2] [3]:

$$
\hat{E}=\hat{K}=i \hbar \frac{\partial}{\partial t}, \hat{p}=-i \hbar \frac{\partial}{\partial x} .
$$

By analogy, Equation (3) can be simply obtained combining Equation (8) with the relation between the relativistic expressions of the kinetic energy and the linear momentum of a free particle traveling at quasi-relativistic speeds:

$$
K=\frac{p^{2}}{\left(\gamma_{V}+1\right) m}, \quad p=\gamma_{V} m V .
$$

Equation (9) can be easily obtained from the following well-known relativistic equations [7] [8]:

$$
\begin{gathered}
E^{2}-m^{2} c^{4}=p^{2} c^{2} \Leftrightarrow\left(E+m c^{2}\right)\left(E-m c^{2}\right)=p^{2} c^{2}, \\
K=E-m c^{2}, E=\gamma_{V} m c^{2} .
\end{gathered}
$$

The Klein-Gordon equation can formally be obtained from the first expression of Equation (10) by assigning the temporal partial derivative operator in Equation (8) to the total relativistic energy $(E)$ of the free particle, which is the sum of its kinetic energy plus the energy associated to the mass of the particle [7] [8]. However, if one chooses to assign this operator to $K$, as it is done when obtaining the Schrödinger equation, then from Equations (9) and (8) follows Equation (3). This is not the customary choice, but in this work instead of simply discharging this option, it is explored the consequences of this natural choice. For instance, a simple substitution of $\psi(x, t)$ given by Equation (6) in Equation (3) results in Equation (9), thus demonstrating that $\psi(x, t)$ given by Equation (6) is a plane wave solution of Equation (3), which phase velocity $V_{p h}=K / p$ is related to the velocity of the particle by the following expression:

$$
V_{p h}=\frac{\gamma_{V}}{\gamma_{V}+1} V
$$

Consequently, $V_{p h}<V<c$, i.e., the plane wave $\psi(x, t)$ given by Equation (6) is subluminal and, as happen for a plane wave solution of the Schrödinger equation, $V_{p h} \sim V / 2$ when $V \ll c$. In contrast, the substitution of $\psi_{K G}(x, t)$ given by Equation (6) in Equation (2) results in Equation (10), thus demonstrating that $\psi_{K G}(x, t)$ given by Equation (6) is a plane wave solution of Equation (2), which phase velocity $V_{K G}=E / p$ is given by the following expression: 


$$
V_{K G}=\frac{\gamma_{V} m c^{2}}{\gamma_{V} m V}=\frac{c^{2}}{V} .
$$

Consequently, $\psi_{K G}(x, t)$ is superluminal because $V_{K G}>c$. Equations (5) and (6) suggest that the time-independent equations corresponding to Equations (2) and (3) are equal. In fact, looking for solutions of the form $X(x) T(t)$ of Equations (1), (2), and (3), where $T(t)=\mathrm{e}^{-\frac{i}{\hbar} K t}$ for Equations (1) and (3) but $T(t)=\mathrm{e}^{-\frac{i}{\hbar} E t}$ for Equation (3), produces the same time-independent equation in the three cases:

$$
\frac{\mathrm{d}^{2}}{\mathrm{~d} x^{2}} X(x)+\kappa^{2} X(x)=0, \kappa=\frac{p}{\hbar} .
$$

As it will be illustrated below, often $X(x)$ and $\kappa$ are determined solving Equation (14) under adequate boundary conditions; then the possible values of $p$ are determinate from the possible values of $\kappa$. However, the relation between $K$ and $p$ are different for non-relativistic and quasi-relativistic speeds; therefore, the solutions of Equations (1) and (3) have equal spatial dependences but different values of $K$. Also, the relation between $E, K$, and $p$ are different for quasi-relativistic speeds; therefore, the solutions of Equations (2) and (3) have equal spatial dependences but different values of $K$ and $E$. Equations (9) and (10) can be obtained from each other using Equation (11); however, Equation (10) admits solutions with positive and negative energies but $K$ only can be positive in Equation (9). This is in correspondence to the presence of a second-order temporal partial derivative in Equation (2), which determines that Equation (2) has solutions with positive and negative energies [8] [9]. In contrast, there is a first-order temporal partial derivative in Equations (1) and (3). This determines that Equations (1) and (3) only have solutions with positive energies. It is straightforward to show that Equation (5) can be obtained from Equations (11) and (6). Equation (5) gives a simple recipe from obtaining a plane wave solution of Equation (3) from a plane wave solution of Equation (2) with positive energy and vice versa.

\section{Probability Density and Galilean Invariance}

Due to the formal similitude between Equation (3) and Equation (1), one can demonstrate that a probability continuity equation can be associated to the solutions of the Grave de Peralta equation in the same way that it is done for the Schrödinger equation [1] [2] [3] [4]. In short, one can associate a probability density $\rho(x, t)$ to a normalized solution of Equation (3) in the following way:

$$
\rho(x, t)=\psi^{*}(x, t) \psi(x, t), \int_{-\infty}^{+\infty} \rho(x, t) \mathrm{d} x=1 .
$$

The probability density corresponding to the Schrödinger equation is well-defined when both $\psi /(2 m)$ and $\psi^{*} /(2 m)$ tend to zero when $|x|$ is very large [1]. Similarly, provided that $V^{2}$ and $\gamma_{V}$ are constant, it can be shown than $\rho(x, t)$ defined by Equation (15) is well-defined when both $\psi /\left[\left(\gamma_{V}+1\right) m\right]$ and 
$\psi^{*} /\left[\left(\gamma_{V}+1\right) m\right]$ tend to zero when $|x|$ is very large, which is a less restrictive condition when $\gamma_{V} \gg 1$ than the one required for the Schrödinger equation. The rate of the temporal variation of $\rho(x, t)$ is then given by the following expression:

$$
\frac{\partial}{\partial t} \rho=\frac{\partial \psi^{*}}{\partial t} \psi+\psi^{*} \frac{\partial \psi^{*}}{\partial t} .
$$

The temporal derivatives of $\psi$ and $\psi^{*}$ in Equation (16) can be substituted by expressions containing spatial derivatives of $\psi$ and $\psi^{*}$ by using Equation (3) and its complex conjugate equation. In this way Equation (16) can be transformed in the following one:

$$
\frac{\partial}{\partial t} \rho=\frac{\hbar}{\left(\gamma_{V}+1\right) m i}\left(\psi \frac{\partial^{2} \psi^{*}}{\partial x^{2}}-\psi^{*} \frac{\partial^{2} \psi}{\partial x^{2}}\right) .
$$

But [1]:

$$
\psi \frac{\partial^{2} \psi^{*}}{\partial x^{2}}-\psi^{*} \frac{\partial^{2} \psi}{\partial x^{2}}=\frac{\partial}{\partial x}\left(\psi \frac{\partial \psi^{*}}{\partial x}-\psi^{*} \frac{\partial \psi}{\partial x}\right)
$$

Then using Equation (18) permits to rewrite Equation (17) as the one-dimensional (1D) probability continuity equation [1]:

$$
\frac{\partial}{\partial t} \rho+\frac{\partial}{\partial x} J=0, \quad J=\frac{\hbar}{\left(\gamma_{V}+1\right) m i}\left(\psi^{*} \frac{\partial \psi}{\partial x}-\psi \frac{\partial \psi^{*}}{\partial x}\right) .
$$

Like for the Schrödinger equation [1], it is easy to show that Equation (19) can be generalized to three dimensions (3D). The absence of negative values of $\rho$ and $J$ is a consequence of the absence of a second time derivative in the Equation (3) [8] [9]. This concludes the demonstration that a probability continuity equation can be associated to the solutions of Equation (3) as it is done for the Schrödinger equation. In what follows a qualitative discussion about the Galilean invariance of Equation (3) is presented. A more formal discussion about this topic is presented in Annex A. At a first sight, Equation (3) does not look neither Galilean nor Lorentz invariant. Equation (3) should not be Lorentz invariant because in Equation (3) the temporal and spatial partial derivatives do not have the same order [8] [9]. In contrast, it is well known that Equation (2) is Lorentz invariant [8] [9]. The formal similitude between Equations (3) and (1) suggests that Equation (3) may be Galileo invariant, but there is a problem. A well-defined Equation (3) requires a constant value of $V^{2}$ and $\gamma_{V}$. As it will be illustrated in Sections 4 and 6 , there are very interesting problems where this requirement is fulfilled. For instance, one of these problems is the description of the movement of a massive quantum particle confined in a $1 \mathrm{D}$ box, which is at rest respect to an inertial reference frame $S$. An observer at rest respect $S$ may think about the particle as moving with constant quasi-relativistic speed $(V)$ but changing direction each time the particle bounced in the box's walls. However, a second observer moving parallel to the box with velocity $+V_{o}$ respect to the first observer, but at rest respect to a second inertial reference frame $S$, would see the particle moving 
sometimes with speed $V_{+}^{\prime}$ and sometimes with speed $V_{-}^{\prime}$, where [7] [8]:

$$
V_{ \pm}^{\prime}=\frac{ \pm V-V_{O}}{1-\frac{ \pm V V_{O}}{c^{2}}}
$$

Thus, the second observer would not find well-defined the value of $V^{2}$ and $\gamma_{V^{\prime}}$ that should be introduced in Equation (3). However, at quasi-relativistic particle's speeds $V_{+}^{\prime} \sim V_{-}^{\prime} \sim V$ when $V_{o} \ll V$. Consequently, at quasi-relativistic particle's speeds when $V_{o} \ll V$, both observers will see the particle moving with (almost) the same values of $V^{2}$ and $\gamma_{V^{*}}$ Moreover, in this quasi-relativistic limit $p^{\prime}$ $\sim p$ and $K^{\prime} \sim K$. Consequently, both observers will agree in that they should solve Equation (3) for finding the possible quantum states of the massive particle moving at quasi-relativistic speeds inside of the $1 \mathrm{D}$ box. i.e., Equation (3) is Galilean invariant. Nevertheless, as it will be shown below, Equation (3) can be used for solving quasi-relativistic quantum problems.

\section{Infinite Rectangular Well}

An important but simple problem often solved in quantum mechanics textbook is a particle moving inside an infinite rectangular well at speeds much smaller than $c$ [2] [3] [4] [5]. Using Equation (3), this problem can be solved for quasi-relativistic speeds following similar procedures than in the quantum mechanics textbooks for $V \ll c$ [2] [3] [4] [5]. One should look for a wavefunction that is identically null outside of the well, null at $x=0$ and $x=L$, and satisfies Equation (3) in the interval $0<x<L$. Solving Equation (3) means finding the quantum states of a free particle with constant values of $K$. But due to Equation (9), a free particle moving with constant kinetic energy must have constant value of $V^{2}$ and vice versa; therefore, the solutions of Equation (3) correspond to quantum states of a particle moving with a constant value of $V^{2}$. Separating variables and substituting in Equation (3) results:

$$
\begin{gathered}
\psi(x, t)=X(x) \mathrm{e}^{\frac{i}{\hbar} K t}, \\
\frac{\mathrm{d}^{2}}{\mathrm{~d} x^{2}} X(x)=-\kappa^{2} X(x)=0, \kappa=\sqrt{\frac{\left(\gamma_{V}+1\right) m K}{\hbar^{2}}}, X(0)=X(L)=0 .
\end{gathered}
$$

Looking for solutions of Equation (22) corresponding to constant values of $K$ and $V^{2}$, one can find that:

$$
\psi_{n}(x, t)=X_{n}(x) \mathrm{e}^{\frac{i}{\hbar} K_{n} t}, \kappa=\frac{n \pi}{L}, n=1,2, \cdots
$$

where:

$$
\begin{aligned}
& X_{n}(x)=\sqrt{\frac{2}{L}} \sin \left(\frac{n \pi}{L} x\right) \\
& K_{n}=n^{2} \frac{h^{2}}{\left(\gamma_{V_{n}}+1\right) m(L / 2)^{2}} .
\end{aligned}
$$


From Equation (24) follows that the spatial dependence of $\psi_{n}(x, t)$ coincide with the spatial dependence of the wavefunction calculated using the Schrödinger equation [2] [3] [4] [5]. As expected, Equation (25) gives the know values of the particle's energies at low speeds when $\gamma_{V} \sim 1$ [2] [3] [4] [5]. From Equation (25) and the relativistic equation, $K=\left(\gamma_{V}-1\right) m c^{2}$, follow:

$$
\gamma_{V_{n}}^{2}=1+n^{2}\left(\frac{2 \lambda_{C}}{L}\right)^{2}
$$

where $\lambda_{C}=h /(m c)$ is the Compton wavelength [7] [8]. Equation (26) gives $\gamma_{V}^{2}=2$ when $n=1$ and $L=2 \lambda_{\dot{O}}$ evaluating for these values Equation (25) results in $K_{1} \sim 0.4 \mathrm{mc}^{2}$, which is smaller than the value $K_{1} \sim 0.5 \mathrm{mc}^{2}$ that would be obtained, using the Schrödinger equation, for the ground state energy of a particle of mass $m$ confined in an infinite rectangular well of length $L=2 \lambda_{C}$. Moreover, this result is precise because the calculated energy of the ground state is clearly quasi-relativistic. In contrast, Equation (26) gives $\gamma_{V}^{2}=5$ when $n=1$ and $L=\lambda_{\dot{C}}$ evaluating for these values Equation (25) results in $K_{1} \sim 1.2 \mathrm{mc}^{2}$. The number of particles may not be constant at these energies. This result for a $1 \mathrm{D}$ infinite rectangular well can easily be extended to the $3 \mathrm{D}$ infinite rectangular well as it is done for the Schrödinger equation [4] [5]. Consequently, Equation (3) establishes a fundamental connection between quantum mechanics and especial theory of relativity: no single particle with mass can be confined in a volume much smaller than $\left(\lambda_{C}\right)^{3}$ because when this occurs, $K>m c^{2}$ and the number of particles may not be constant anymore; therefore, a single point-particle with mass cannot exist. Point-particles with mass can only exist in fully relativistic quantum field theories where the number of particles is not constant. This is true for an electron, a quark, and probably may also be true for a black hole and the whole universe at the beginning of the Big Bang. This is consistent, for instance, with the confinement of an electron in the Hydrogen atom because for an electron $\lambda_{C e} \sim 2.4 \times 10^{-3} \mathrm{~nm}$, which is $\sim 20$ times smaller than the radius of the Hydrogen atom, $r_{B} \sim 5.3 \times 10^{-2} \mathrm{~nm}$ [1] [2] [3] [4] [5]. Combining Equations (25) and (26) allows for rewritten Equation (25) in the following way:

$$
K_{n}=n^{2} \frac{h^{2}}{\left[1+\sqrt{1+\left(\frac{2 n \lambda_{C}}{L}\right)^{2}}\right] m\left(\frac{L}{2}\right)^{2}} .
$$

When $L \gg 2 n \lambda_{C}$, Equation (27) gives the know values of the energies calculated using the Schrodinger equation for a particle in an infinite well [2] [3] [4] [5]. However, in general, the values of $K_{n}$ calculated using Equation (27) are smaller than the ones calculated using the Schrödinger equation. This in excellent correspondence with more involved numerical results obtained solving the Dirac equation for the 1D infinite rectangular well [10]. Moreover, and more significant for experiments, the differences in energies between different energy levels are slightly different when obtained using Equations (1) and (3). 


\section{Superposition Principle}

Besides allowing to obtain precise quasi-relativistic solutions of several interesting problems, like tunneling through a barrier and other problems with piecewise constant potentials, following similar procedures than in the quantum mechanics textbooks for $V \ll c$ [1] [2] [3] [4] [5], Equation (3) may describe a new physics. The Schrödinger equation is linear, this means, for instance, that if $\psi_{s c h 1}(X, t)$ and $\psi_{s c h 2}(X, t)$ are two solutions of Equation (1) for a particle in an infinite rectangular well corresponding to different values of $V^{2}$, then the wavefunction $\psi_{\text {Sch }}(x, t)=a \psi_{\text {Sch } 1}(x, t)+b \psi_{\text {sch } 2}(x, t)$, where $a$ and $b$ are complex numbers such that $|a|+|b|^{2}=1$, is also a solution of Equation (1). $\psi_{s c h}(x, t)$ represents a legitime possible state of a particle in an infinite well. The superposition state represented by $\psi_{s c h}(X, t)$ is often interpreted as a state where the particle is neither in the state $\psi_{\text {schl }}(x, t)$ where the kinetic energy is $K_{1}$ nor in the state $\psi_{\text {sch } 2}(x, t)$ where the kinetic energy is $K_{2}$, but somehow the particle is simultaneously in both states. The existence of superposition states like $\psi_{S c h}(x, t)$ is then a fundamental consequence of the linearity of Equation (1) with no classical counterpart. This exemplifies the weirdness of quantum mechanics [6] [11]. Moreover, the superposition state $\psi_{s c h}(x, t)$ represent a qubit, concept that is at the heart of current attempts to demonstrate a practical quantum computer [11] [12]. In contrast to the Schrödinger equation, Equation (3) is not linear. If $\psi_{1}(x$, $t)$ and $\psi_{2}(x, t)$ are two solutions of Equation (3) for a particle in a rectangular infinite well corresponding to different values of $V^{2}$, then strictly they are not solutions of the same Equation (3) but of slightly different Equations (3) with different values of $\gamma_{V}$. Moreover, $\psi(x, t)=a \psi_{1}(x, t)+b \psi_{2}(x, t)$ is not a solution of any Equation (3). Consequently, if the Grave de Peralta equation is a legitime extension of the Schrödinger equation to the quasi-relativistic domain, then the current understanding of the superposition principle in quantum mechanics should be revised because it appears to only be valid when the particle moves at speeds much smaller than $c$. The superposition principle is a corner stone of quantum mechanics; therefore, one could be interested in saving the superposition principle by stretching the meaning of "solution of Equation (3)", such that " $\psi(x, t)=a \psi_{1}(x, t)+b \psi_{2}(x, t)$ is a solution of Equation (3)" means that there is a set formed by several slightly different Equations (3) and $a \psi_{1}(x, t)$ and $b \psi_{2}(x, t)$ are solutions of a slightly different Equation (3) from this set, corresponding to a different value of $V^{2}$ each. For instance, strictly speaking, Equations (23), (24), and (27) give the solutions of a set of Equations (3) for the infinite rectangular well. This is somehow related with Section 3 discussion about the Galilean invariance of Equation (3). Strictly speaking, two observers slowly traveling with constant velocity respect to each other should resolve a set of Equations (3) which values of $\gamma_{V}$ are contained in a narrow continuous interval. The adoption of Equation (3) as a valid description of the quantum states of a massive free particle then breaks with the longstanding tradition of describing the dynamics of a physical system using a single equation. The second Newton law and the 
Schrödinger equation are examples of this tradition. The future of the particle's wave function is determined by the Schrödinger equation and the initial conditions are the only source of indeterminacy. In contrast, the dynamics of a particle is described by a whole set of similar Equations (3), which introduces a new source of indeterminacy in the future of the particle's wave function. This may be a welcome development for the understanding of the weirdness of quantum mechanics.

Alternatively, the non-linearity of Equation (3) suggests that $\psi_{1}(x, t)$ and $\psi_{2}(x$, $t$ ) could be understood as corresponding to two different phases-of-a-system which are described by a different equation each. $\psi(x, t)$, which is not a solution of Equation (3), describes them a state of the system where no one of these two phases exists but where somehow, when a set of identical measurements is done on a system which is prepared in the state $\psi(x, t)$ each time, then a fast transition of the system is induced by the measurement and the system randomly transits either to the phase represented by $\psi_{1}(x, t)$ with probability $|a|^{2}$ or to the phase represented by $\psi_{2}(x, t)$ with probability $|b|^{2}$. In this description, the state of the system represented by $\psi(x, t)$ must be different from a state where a mixture of the phases $\psi_{1}(x, t)$ and $\psi_{2}(x, t)$ actually exist. For instance, let's assume that $\psi_{1}(x$, $t)$ and $\psi_{2}(x, t)$ are two solutions, of the set of Equations (3) for the infinite rectangular well, with kinetic energies given by $K_{1}$ and $K_{2}$, respectively. Loosely borrowing the words "superheated" and "supercooled" from possible phase transitions in liquids, one could say that the state $\psi(x, t)=a \psi_{1}(x, t)+b \psi_{2}(x, t)$ corresponds to a state of the system which is not a solution of any Equation (3). The state $\psi(x, t)$ could be formed by superheating the state $\psi_{1}(x, t)$ or by supercooling the state $\psi_{2}(x, t)$. When a set of $N$ measurements is done on the system in the state $\psi(x, t),|a|^{2} N$ times a fast system's transition occurs from the superheated state to the state $\psi_{1}(x, t)$, and $|b|^{2} N$ times the transition occurs from the supercooled state to the state $\psi_{2}(x, t)$. This point of view may motivate the search for the unknown equation for which $\psi(x, t)$ is a solution.

\section{Quasi-Relativistic Quantum Rotor}

Courses of Quantum Mechanics often include how to solve the Schrödinger equation for a quantum rigid rotator, which in general is a quantum particle moving with constant speed in a sphere. Therefore, all the kinetic energy of a quantum rigid rotator is rotational. Instances of the quantum rigid rotor appear when describing the relative movement between two particles forming a system like a diatomic molecule (neglecting vibrations) [5]. The 3D Schrödinger equation for a particle moving in a central potential $\Phi(r)$ is given by the following equation [1] [2] [3] [4] [5]:

$$
i \hbar \frac{\partial}{\partial t} \psi_{S c h}(\boldsymbol{r}, t)=-\frac{\hbar^{2}}{2 m} \nabla^{2} \psi_{S c h}(\boldsymbol{r}, t)+\Phi(r) \psi_{S c h}(\boldsymbol{r}, t) .
$$

Which natural extension to quasi-relativistic speeds is the following equation: 


$$
i \hbar \frac{\partial}{\partial t} \psi_{S c h}(\boldsymbol{r}, t)=-\frac{\hbar^{2}}{2 m} \nabla^{2} \psi_{S c h}(\boldsymbol{r}, t)+\Phi(r) \psi_{S c h}(\boldsymbol{r}, t) .
$$

In spherical coordinates $\boldsymbol{r}=(r, \theta, \varphi)$, and the Laplacian operator in Equations (28) and (29) is defined in the following way [1] [2] [3] [4] [5]:

$$
\nabla^{2} \psi=\frac{1}{r} \frac{\partial^{2}}{\partial r^{2}}(r \psi)+\frac{1}{r^{2}} \nabla_{\theta, \varphi}^{2} \psi
$$

Where:

$$
\nabla_{\theta, \varphi}^{2}=\frac{1}{\sin \theta} \frac{\partial}{\partial \theta}\left(\sin \theta \frac{\partial}{\partial \theta}\right)+\frac{1}{\sin ^{2} \theta} \frac{\partial^{2}}{\partial \varphi^{2}} .
$$

Using Equations (30) and (31) permits to rewrite Equation (29) in the following way:

$$
i \hbar \frac{\partial}{\partial t} \psi=-\frac{\hbar^{2}}{\left(\gamma_{V}+1\right) m r} \frac{\partial^{2}}{\partial r^{2}}(r \psi)-\frac{\hbar^{2}}{\left(\gamma_{V}+1\right) m r^{2}} \nabla_{\theta, \varphi}^{2} \psi+\Phi(r) \psi .
$$

The rotational kinetic energy of a quantum rigid rotator is given by the second term in the right size of Equation (32); therefore, the first term in the right size of Equation (32) vanishes for a quantum rigid rotator [5]. In addition, $r=r_{S}$ and $\Phi(r)=\Phi\left(r_{S}\right)$ are constants because the radius of the sphere containing the particle trajectory $\left(r_{S}\right)$ is constant; therefore, choosing $\Phi\left(r_{S}\right)=0$, and introducing the moment of inertia of a rotating mass $I=m r_{S}^{2}$, reduces Equation (32) to the following expression for a quasi-relativistic quantum rigid rotator:

$$
i \hbar \frac{\partial}{\partial t} \psi(\theta, \varphi)=-\frac{\hbar^{2}}{\left(\gamma_{V}+1\right) I} \nabla_{\theta, \varphi}^{2} \psi(\theta, \varphi) .
$$

Equation (33) can be solved looking for a separable-variable solution of the following form:

$$
\psi(\theta, \varphi, t)=\Omega(\theta, \varphi) \mathrm{e}^{\frac{i}{\hbar} K t} .
$$

Then, substituting Equation (34) in Equation (33), results in the well-known equation for the spherical harmonic functions [1] [2] [3] [4] [5]:

$$
\nabla_{\theta, \varphi}^{2} \Omega(\theta, \varphi)+\eta \Omega(\theta, \varphi)=0 .
$$

With:

$$
\eta=\frac{\left(\gamma_{V}+1\right) I}{\hbar^{2}} K
$$

where $K$ is the quasi-relativistic kinetic energy of the rotor. Consequently, the normalized solution of Equation (35) satisfying the appropriated boundary conditions is given by the following expressions [1] [2] [3] [4] [5]:

$$
\Omega_{l, m}(\theta, \varphi) \propto Y_{l}^{(m)}(\theta, \varphi) ; \eta=l(l+1) ; l=0,1,2, \cdots ; m=-l,-l+1, \cdots, 0,1, \cdots, l .
$$

where $Y_{l}^{(m)}$ are the spherical harmonic functions [1] [2] [3] [4] [5]. Therefore, the quasi-relativistic kinetic energy is given by the following expression: 


$$
K_{l}=\frac{\hbar^{2}}{\left(\gamma_{V_{l}}+1\right) I} l(l+1)=\frac{h^{2}}{\left(\gamma_{V_{l}}+1\right) m L_{C}^{2}} l(l+1) .
$$

where $L_{C}=2 \pi r_{S}$ is the maximum length of a circle contained in the sphere where the particle moves. From Equation (37) follows that the spatial dependence of $\psi_{l, m}$ coincide with the spatial dependence of the wavefunction calculated using the Schrödinger equation [5]. As expected, Equation (38) gives the know values of the particle's energies at low speeds when $\gamma_{V} \sim 1$ [5]. From Equation (38) and the relativistic equation, $K=\left(\gamma_{V}-1\right) m c^{2}$, follow:

$$
\gamma_{V_{l}}^{2}=1+\left(\frac{\lambda_{C}}{L_{C}}\right)^{2} l(l+1) .
$$

Equation (39) gives $\gamma_{V}^{2}=3$ when $I=1$ and $L_{C}=\lambda_{\dot{C}}$ evaluating for these values Equation (38) results in $K_{1} \sim 0.7 \mathrm{mc}^{2}$, which is smaller than the value $K_{1} \sim$ $m c^{2}$ that would be obtained, using the Schrödinger equation, for the state with minimum non-zero angular momentum $(I=1)$ of a quantum rotor with $L_{C}=\lambda_{C}$ [5]. Moreover, this result is precise because the calculated energy $\left(K_{1} \sim 0.7 \mathrm{mc}^{2}\right)$ is quasi-relativistic. In contrast, Equation (39) gives $\gamma_{V}^{2}=9$ when $I=1$ and $L_{C}=$ $\lambda_{d} 2$; evaluating for these values Equation (38) results in $K_{1}=2 m c^{2}$. The number of particles may not be constant at this energy. Consequently, Equation (3) also establishes the following fundamental connection between quantum mechanics and especial theory of relativity: there is a stable orbit with minimum length that a quantum particle of mass $m$, moving with constant non-zero speed in a sphere, can have. This length is equal to the Compton wavelength associated to the particle's mass. Combining Equations (38) and (39) allow for rewritten Equation (38) in the following way:

$$
K_{l}=\frac{h^{2}}{\left[1+\sqrt{1+\left(\frac{\lambda_{C} \sqrt{l(l+1)}}{L_{C}}\right)^{2}}\right] m L_{C}^{2}} l(l+1) .
$$

When $L_{C} \gg \sqrt{l(l+1)} \lambda_{C}$, Equation (40) gives the know values of the energies calculated using the Schrödinger equation for a non-relativistic quantum rotor [5]. However, in general, the values of $K_{l}$ calculated using Equation (40) are smaller than the ones calculated using the Schrödinger equation. Moreover, and more significant for experiments, the differences in energies between different energy levels are slightly different when obtained using Equations (1) and (3).

\section{Conclusion}

Relativistic quantum mechanics has evolved a lot since 1925, when Erwin Schrödinger played with the Klein-Gordon equation but decided not to publish what he found and then, settled for publishing his finding about the today famous Schrödinger equation. Nevertheless, the existence of the quasi-relativistic Schrödinger-like equation discussed here should be considered the discovery of 
a hidden gem. At low particle's speeds, the proposed Grave de Peralta equation (Equation (3)) clearly coincides with the Schrödinger equation. Equation (3) is Galilean invariant for observers traveling at low speeds respect to each other, and a positive probability density can be defined for this equation by analogy of how it is defined for the Schrödinger equation. The plane wave solutions of Equation (3) are subluminal and are related through Equation (5) with the plane wave solutions with positive energies of the Klein-Gordon equation. From a practical point of view Equation (3) has a clear scholastic value. Moreover, as it was shown in this work, Equation (3) can be used for obtaining precise quasi-relativistic solutions of very interesting problems at energies smaller than $m c^{2}$.

\section{Acknowledgements}

The author recognizes the valuable discussions with Ankit Pandey from the Department of Chemistry at Texas Tech University and Arquimedes Ruiz-Columbié from the National Wind Institute at Texas Tech University.

\section{Conflicts of Interest}

The author declares no conflicts of interest regarding the publication of this paper.

\section{References}

[1] Bohm, D. (1964) Quantum Theory. 11th Edition, Prentice Hall, Upper Saddle River.

[2] Davydov, A.S. (1965) Quantum Mechanics. Pergamon Press, New York.

[3] Merzbacher, E. (1970) Quantum Mechanics. 2nd Edition, J. Wiley \& Sons, New York.

[4] Griffiths, D.J. (1995) Introduction to Quantum Mechanics. Prentice Hall, Upper Saddle River.

[5] Levine, I.N. (2014) Quantum Chemistry. 7th Edition, Pearson Education, New York.

[6] Home, D. (1997) Conceptual Foundations of Quantum Physics: An Overview from Modern Perspectives. Plenum Press, New York.

[7] Jackson, J.D. (1975) Classical Electrodynamics. 2nd Edition, J. Wiley \& Sons, New York.

[8] Strange, P. (1998) Relativistic Quantum Mechanics: With Applications in Condensed Matter and Atomic Physics. Cambridge University Press, New York. https://doi.org/10.1017/CBO9780511622755

[9] Greiner, W. (1990) Relativistic Quantum Mechanics: Wave Equations. Spring-Verlag, New York. https://doi.org/10.1007/978-3-662-02634-2

[10] Alberto, P., Fiolhais, C. and Gil, V.M.S. (1996) European Journal of Physics, 17, 19-24. https://doi.org/10.1088/0143-0807/17/1/004

[11] Nielsen, M.A. and Chuang, I.L. (2000) Quantum Computation and Quantum Information. Cambridge University Press, Cambridge.

[12] DiVincenzo, D.P. (1995) Science, 270, 255-261. https://doi.org/10.1126/science.270.5234.255 


\section{Annex A: Lorentz or Galilean Invariance?}

It is well-known that Equation (10) is Lorentz invariant [7] [8] [9]. The 3D version of Equation (10) can be rewritten in a covariant form in the following way [7] [8] [9]:

$$
p^{\mu} p_{\mu}=\frac{E^{2}}{c^{2}}-\boldsymbol{p} \cdot \boldsymbol{p}=m^{2} c^{2}, \quad p^{\mu}=\left\{\frac{E}{c}, p_{x}, p_{y}, p_{z}\right\} .
$$

Here covariance means that the module $\left(p^{\mu} p_{\mu}\right)$ of the four-component vector $p^{u}$ is a scalar under Lorentz transformations because $m^{2} c^{2}$ is a scalar under the Lorentz transformations relating the coordinates $\{t, x, y, z\}$, respect to an inertial reference frame $S$, to the coordinates $\left\{t^{\prime}, x^{\prime}, y^{\prime}, z^{\prime}\right\}$, respect to a second inertial reference frame $S^{\prime}$ which is moving with speed $V_{o}$ respect to $S$ in the positive direction of the axis $x$ [7] [8]:

$$
t=\gamma_{V_{o}}\left(t^{\prime}+\frac{V_{o}}{c^{2}} x^{\prime}\right), x=\gamma_{V_{o}}\left(x^{\prime}+V_{o} t^{\prime}\right), \quad y=y^{\prime}, \quad z=z^{\prime} .
$$

Equation (8) permits to associate a partial derivative operator to each component of $p^{\mu}$, thus transforming Equation (A1) in the 3D version of Equation (2) [8] [9]:

$$
\begin{aligned}
& \hat{p}^{\mu} \hat{p}_{\mu} \psi_{K G}(x, y, x, t)=m^{2} c^{2} \psi_{K G}(x, y, x, t), \\
& \hat{p}^{\mu}=\left\{i \hbar \frac{\partial}{\partial c t}, i \hbar \frac{\partial}{\partial x}, i \hbar \frac{\partial}{\partial y}, i \hbar \frac{\partial}{\partial z}\right\} .
\end{aligned}
$$

The operator $\hat{p}^{\mu}$ is a Lorentz-invariant four-component vector because $\psi_{K G}(x, y, z, t)$, the wavefunction of a particle with spin-0, must be a Lorentz-scalar [8] [9], and because the four quantities formed by differentiation of a Lorentz-scalar respect to the components of a Lorentz-invariant four-vector transform as a four-component Lorentz invariant vector [7]. Therefore, the left term of Equation (A3) transform under Lorentz transformations as the module of a four-component Lorentz-invariant vector; i.e., as a Lorentz-scalar. Consequently, provided that $\psi_{K G}(x, y, z, t)$ is a scalar under Lorentz transformations, Equation (A3) is relativistic covariant; i.e., both sides of the equation transform under Lorentz transformations as Lorentz-scalars. Equation (A3) can then be directly written in differential form in the same way in both $S$ and $S$ ' references frames:

$$
\begin{aligned}
& \frac{1}{c^{2}} \frac{\partial^{2}}{\partial t^{2}} \psi_{K G}(\boldsymbol{r}, t)=\nabla^{2} \psi_{K G}(\boldsymbol{r}, t)-\frac{m^{2} c^{2}}{\hbar^{2}} \psi_{K G}(\boldsymbol{r}, t), \\
& \frac{1}{c^{2}} \frac{\partial^{2}}{\partial t^{\prime 2}} \psi_{K G}^{\prime}\left(\boldsymbol{r}^{\prime}, t^{\prime}\right)=\nabla^{\prime 2} \psi_{K G}^{\prime}\left(\boldsymbol{r}^{\prime}, t^{\prime}\right)-\frac{m^{2} c^{2}}{\hbar^{2}} \psi_{K G}^{\prime}\left(\boldsymbol{r}^{\prime}, t^{\prime}\right) .
\end{aligned}
$$

where $\boldsymbol{r}=(x, y, z)$ is a $3 \mathrm{D}$ spatial vector is rectangular coordinates. Evidently, Equation (A4) is a 3D version of Equation (2). Therefore, a 3D version of Equation (6) is a plane wave solution of Equation (A4), which is a Lorentz-scalar because the wave's phase can be rewritten as the scalar product of two Lorentz-invariant four-components vectors [7] [8] [9]: 


$$
\psi_{K G}(\boldsymbol{r}, t)=\mathrm{e}^{\frac{i}{\hbar}(\boldsymbol{p} \cdot \boldsymbol{r}-E t)}=\mathrm{e}^{\frac{i}{\hbar}\left(-p^{\mu} x_{\mu}\right)}, x^{\mu}=\{c t, x, y, z\} .
$$

Therefore, $\psi_{K G}^{\prime}\left(x^{\prime}, y^{\prime}, z^{\prime}, t^{\prime}\right)$ is the boosted wavefunction:

$$
\psi_{K G}^{\prime}\left(x^{\prime}, y^{\prime}, z^{\prime}, t^{\prime}\right)=\mathrm{e}^{\frac{i}{\hbar}\left(p^{\prime} \cdot r^{\prime}-E^{\prime} t^{\prime}\right)} .
$$

In other words, $\psi_{K G}^{\prime}\left(x^{\prime}, y^{\prime}, z^{\prime}, t^{\prime}\right)$ can be obtained from $\psi_{\mathrm{KG}}(x, y, z, t)$ by making the formal substitutions $x \rightarrow x\left(x^{\prime}, t\right), y \rightarrow y^{\prime}, z \rightarrow z^{\prime}$, and $t \rightarrow t\left(x^{\prime}, t^{\prime}\right)$ using Equation (A2). In addition, the linear momentum and total energy of the particle should be boosted by substituting $p \rightarrow p^{\prime}$ and $E \rightarrow E^{\prime}$. This ends the discussion about the Lorentz invariance of the Klein-Gordon equation. One can then try to demonstrate the relativistic covariance of Equation (3) following similar steps than for the demonstration of the relativistic covariance of the Klein-Gordon equation. Looking for a solution of the $3 \mathrm{D}$ version of Equation (3):

$$
i \hbar \frac{\partial}{\partial t} \psi(\boldsymbol{r}, t)=-\frac{\hbar^{2}}{\left(\gamma_{V}+1\right) m} \nabla^{2} \psi(\boldsymbol{r}, t),
$$

Such that:

$$
\psi(\boldsymbol{r}, t)=\mathrm{e}^{\frac{i}{\hbar}(\boldsymbol{p} \cdot \boldsymbol{r}-K t)}, \psi^{\prime\left(\boldsymbol{r}^{\prime}, t^{\prime}\right)}=\mathrm{e}^{\frac{i}{\hbar}\left(\boldsymbol{p}^{\prime} \cdot \boldsymbol{r}^{\prime}-K^{\prime} t^{\prime}\right)},
$$

Would require that:

$$
\psi(\boldsymbol{r}, t)=\mathrm{e}^{\frac{i}{\hbar}(\boldsymbol{p} \cdot \boldsymbol{r}-K t)}=\mathrm{e}^{\frac{i}{\hbar}\left(-\beta^{\mu} x_{\mu}\right)}, x^{\mu}=\{c t, x, y, z\} .
$$

But Equation (A9) would imply the following relation between $K$ and $p$.

$$
\beta^{\mu} p_{\mu}=\frac{K^{2}}{c^{2}}-\boldsymbol{p} \cdot \boldsymbol{p}=0, \beta^{\mu}=\left\{\frac{K}{c}, p_{x}, p_{y}, p_{z}\right\}, K^{2}=p^{2} c^{2} .
$$

This relation is only correct for relativistic massless particles (photons). For a particle with non-zero mass, the correct relativistic relation between $K$ and $p$ is given by Equation (9). This demonstrates that Equations (3) and $A(7)$ are not Lorentz invariant. Qualitative arguments about the Galilean invariance of Equation (3) were given in Section 3; therefore, one should expect that Equation (3) is approximately Galileo invariant when the observers move respect to each other at much smaller speeds than the quasi-relativistic speed of the particle. One can then try to demonstrate the Galilean invariance of Equation (3) following similar steps than for the demonstration of the Galilean invariance of the Schrödinger equation [6]. Consequently, when $V_{o} \ll V$ and $K \sim m c^{2}$, if an observer at rest respect to S' find that:

$$
\psi^{\prime}\left(x^{\prime}, t^{\prime}\right)=\mathrm{e}^{\frac{i}{\hbar}\left(p^{\prime} x^{\prime}-K^{\prime} t^{\prime}\right)}
$$

Is a solution of the equation:

$$
i \hbar \frac{\partial}{\partial t^{\prime}} \psi^{\prime}\left(x^{\prime}, t^{\prime}\right)=-\frac{\hbar^{2}}{\left(\gamma_{V^{\prime}}+1\right) m} \frac{\partial^{2}}{\partial x^{\prime 2}} \psi^{\prime}\left(x^{\prime}, t^{\prime}\right) .
$$

Then, if Equation (A12) is Galilean invariant, an observer at rest respect to S 
should be able to find that the following plane wave:

$$
\psi(x, t)=\psi^{\prime}\left(x^{\prime}, t^{\prime}\right) \mathrm{e}^{i \varepsilon(x, t)}=\mathrm{e}^{\frac{i}{\hbar}\left(p^{\prime} x^{\prime}-K^{\prime} t^{\prime}\right)} \mathrm{e}^{i \varepsilon(x, t)},
$$

Is a solution of Equation (3) [6]. In Equation (A13), $\psi^{\prime}\left(x^{\prime}, t^{\prime}\right)$ should be explicitly rewritten as $\psi^{\prime}(x, t)$ by substituting the variables $x^{\prime}, t^{\prime}$ by the variables $x, t$ using the Galileo transformations [6]:

$$
t=t^{\prime}, \quad x=x^{\prime}+V_{o} t^{\prime}
$$

In Equation (A13), $\varepsilon(x, t)$ have a double function. First, it should make $\psi(x, t)$ a Galilean boosted version of $\psi^{\prime}\left(x^{\prime}, t^{\prime}\right)$, i.e.:

$$
\psi(x, t)=\mathrm{e}^{\frac{i}{\hbar}\left[p^{\prime}\left(x-V_{0} t\right)-K^{\prime} t\right]} \mathrm{e}^{i \varepsilon(x, t)}=\mathrm{e}^{\frac{i}{\hbar}(p x-K t)} .
$$

The equation in the variables $x$ and $t$ that results, after using Equation (A14) for transforming the differential operators of Equation (A12), do not need to be equal to Equation (3). Therefore, the second function of $\varepsilon(x, t)$ is to guarantee that $\psi(x, t)$ given by Equations (A13) and (A15) satisfies Equation (3). If there is a function $\varepsilon(x, t)$ satisfying these two requirements, then $\psi(x, t)$ and $\psi^{\prime}\left(x^{\prime}, t^{\prime}\right)$ both satisfy the same equation and both have equal square module values; therefore, both described the same physical reality [6]. i.e., Equation (3) would be Galilean invariant. It can be shown that for the Schrödinger equation, $\varepsilon_{S c h}(X$, $t$ ) is given by the following equation [6]:

$$
\varepsilon_{\text {Sch }}(x, t)=\frac{1}{\hbar}\left(m V_{o} x-\frac{1}{2} m V_{o}^{2} t\right) .
$$

Therefore, $\psi_{s c h}(x, t)$ is a boosted version of $\psi_{S c h}^{\prime}\left(x^{\prime}, t^{\prime}\right)$ because for a non-relativistic particle:

$$
\begin{aligned}
& \frac{i}{\hbar}\left(p^{\prime} x^{\prime}-K^{\prime} t^{\prime}\right)+\varepsilon_{S c h}(x, t) \\
& =\frac{i}{\hbar}\left[p^{\prime}\left(x-V_{o} t\right)-\frac{p^{\prime 2}}{2 m} t\right]+\frac{1}{\hbar}\left(m V_{o} x-\frac{1}{2} m V_{o}^{2} t\right) \\
& =\frac{i}{\hbar}\left[\left(p^{\prime}+m V_{o}\right) x-\frac{\left(p^{\prime}+m V_{o}\right)^{2}}{2 m} t\right] \\
& =\frac{i}{\hbar}\left[p x-\frac{p^{2}}{2 m} t\right]=\frac{i}{\hbar}(p x-K t) .
\end{aligned}
$$

In addition $\psi_{s c h}(x, t)$ satisfies Equation (1) [6]. What follows is the demonstration that Equation (3) is approximately Galileo invariant when $V_{o} \ll V$, and $K$ $\sim m c^{2}$. One should find a function $\varepsilon(x, t)$ for Equation (A12) that satisfies the two requirements discussed above. First, using the Galilean relations given by Equation (A14), the differential operators in the variables $x^{\prime}$ and $t^{\prime}$ in Equation (A12) transform to the following differential operators in the variables $x$ and $t$ [6]:

$$
\frac{\partial}{\partial t^{\prime}}=\frac{\partial t}{\partial t^{\prime}} \frac{\partial}{\partial t}+\frac{\partial x}{\partial t^{\prime}} \frac{\partial}{\partial x}=\frac{\partial}{\partial t}+V_{o} \frac{\partial}{\partial x}, \frac{\partial t}{\partial x^{\prime}}=\frac{\partial t}{\partial x^{\prime}} \frac{\partial}{\partial t}+\frac{\partial x}{\partial x^{\prime}} \frac{\partial}{\partial x}=\frac{\partial}{\partial x} .
$$


After transforming Equation (A12) using Equation (A18) and making $\gamma_{V} \sim \gamma_{V}$ because $V_{o} \ll V$, and $K \sim m c^{2}$, one can propose:

$$
\psi(x, t)=\psi^{\prime}(x, t) \mathrm{e}^{i \varepsilon(x, t)} .
$$

where $\psi^{\prime}(x, t)$ is $\psi^{\prime}\left(x^{\prime}, t^{\prime}\right)$, i.e., a solution of Equation (A12), after making the substitution $x^{\prime} \rightarrow x^{\prime}(x, t)$, and $t^{\prime} \rightarrow t$ using Equation (A14). Then one can substitute $\psi(x, t)$ given by Equation (A19) in the equation that results after transforming Equation (A12) using Equation (A18). This permits finding out that $\mathcal{E}(x, t)$ must satisfy the following conditions provided $\psi(x, t)$ is a solution of Equation (3):

$$
\frac{2 \hbar}{\left(\gamma_{V}+1\right) m} \frac{\partial \varepsilon}{\partial x}-V_{o}=\frac{\partial^{2} \varepsilon}{\partial x^{2}}=\frac{\hbar}{\left(\gamma_{V}+1\right) m}\left(\frac{\partial \varepsilon}{\partial x}\right)^{2}-V_{o} \frac{\partial \varepsilon}{\partial x}-\frac{\partial \varepsilon}{\partial t}=0
$$

These conditions are very similar to the ones corresponding to the Schrödinger equation [6]. The three conditions given by Equation (A20) determine that $\varepsilon(x, t)$ is given by the following expression:

$$
\varepsilon(x, t)=\frac{1}{\hbar}\left[\frac{1}{2}\left(\gamma_{V}+1\right) m V_{o} x-\frac{1}{4}\left(\gamma_{V}+1\right) m V_{o}^{2} t\right] .
$$

Comparing Equations (A16) and (A21), one realizes that both include a linear momentum term and a kinetic energy term. Equation (A16) includes the non-relativistic expressions $p_{o}=m V_{o}$ and $K_{o}=p_{o}^{2} /(2 m)$. Equation (A24) includes the relativistic expressions $p_{r}=\frac{1}{2}\left(\gamma_{v}+1\right) m V_{o}$ and $K_{r} \sim p_{r}^{2} /\left[\left(\gamma_{v}+1\right) m\right]$. This is because the Schrödinger equation describes a non-relativistic particle but Equation (3) describes a particle moving at quasi-relativistic speeds. Finally, one should check if $\varepsilon(x, t)$ given by Equation (A21) transforms $\psi(x, t)$ in a Galilean boosted version of $\psi^{\prime}\left(x^{\prime}, t^{\prime}\right)$. i.e., one should check if:

$$
\mathrm{e}^{\frac{i}{\hbar}\left[p^{\prime}\left(x-V_{0} t\right)-\frac{p^{\prime 2}}{\left(\gamma_{V}+1\right) m} t\right]} \mathrm{e}^{i \varepsilon(x, t)} \approx \mathrm{e}^{\frac{i}{\hbar}\left[p x-\frac{p^{2}}{\left(\gamma_{V}+1\right) m} t\right]} .
$$

But:

$$
\begin{aligned}
& \frac{i}{\hbar}\left[p^{\prime}\left(x-V_{o} t\right)-\frac{p^{\prime 2}}{\left(\gamma_{V}+1\right) m} t\right]+\frac{1}{\hbar}\left[\frac{1}{2}\left(\gamma_{V}+1\right) m V_{o} x-\frac{1}{4}\left(\gamma_{V}+1\right) m V_{o}^{2} t\right] \\
& =\frac{i}{\hbar}\left\{\left[p^{\prime}+\frac{1}{2}\left(\gamma_{V}+1\right) m V_{o}\right] x-\frac{\left[p^{\prime}+\frac{1}{2}\left(\gamma_{V}+1\right) m V_{o}\right]^{2}}{\left(\gamma_{V}+1\right) m} t\right\} .
\end{aligned}
$$

When $V_{o} \ll V$, and $K \sim m c^{2}$, then $p^{\prime} \sim\left(\gamma_{v}+1\right) m V \gg p_{r}$ and $p^{\prime}+p_{r} \sim p^{\prime} \sim p$. Therefore, Equation (A23) can be approximated to the following expression:

$$
=\frac{i}{\hbar}\left[p x-\frac{p^{2}}{\left(\gamma_{V}+1\right) m} t\right]=\frac{i}{\hbar}(p x-K t) .
$$


Consequently, Equation (3) is Galilean invariant for observers that move at constant but small speed respect to each other. Nevertheless, the Grave de Peralta equation describes a massive free quantum particle moving at quasi-relativistic speeds. 